\title{
Corrosion Casting of the Microvasculature in Normal Limbs and Limbs with Venous Ulceration.
}

\author{
M.N. Phillips, ${ }^{*} * *$ A.M. van Rij, ${ }^{*}$ M. Zhang,** and G.T. Jones*. \\ * Department of Medicine and Surgery, University of Otago, Dunedin 9001, New Zealand \\ ** Department of Anatomy and Structural Biology, University of Otago, Dunedin 9001, New \\ Zealand .
}

Controversy exists regarding the presence of valves in veins less than $2 \mathrm{~mm}$ in diameter.

Recently venous valves have been identified in the small superficial veins of the human lower limb using vascular corrosion casting. The role that these valves have in both normal limbs and limbs with venous ulceration is unclear. This study proposed to assess differences in valve morphology in the microcirculation of normal and diseased limbs, using microvascular corrosion casting.

Five amputated lower limbs with venous disease (CVI class 6; three males, two females; 61 -86 years) were retrogradely filled with resin via the dorsal vein of the foot. Superficial tissue $(20 \times 20 \mathrm{~mm})$ from the gaiter, lower, mid and upper calf were removed and macerated $(15 \%$ $\mathrm{NaOH}$ ). Resin casts were viewed by light and scanning electron microscopy (FIG 1), and compared with similar casts from six normal cadaver limbs (CVI class $0-1$; four males, two females; 59-92 years).

Small venous valves were present in all regions from the normal limbs and limbs with venous ulceration (FIG 2), The valve number, density and size distribution between the normal and diseased limbs were not different. However, the limbs with venous ulceration had a greater density and tortuosity of veins filled with resin compared with normal limbs $\left(153.8\right.$ vessels $/ \mathrm{cm}^{3}$ vs. 15.1 vessels $/ \mathrm{cm}^{3}$ respectively). Consequently, the valves/vein-density was lower in the diseased limbs when compared to the normal ( 0.02 vs. 0.16 respectively). This may reflect microvenous reflux in these diseased limbs.

This study shows, for the first time, that the number, density and size of microvenous valves does not differ between corresponding sites in normal and venous ulcerated limbs. Significantly, the microvalves of the venous ulcerated limbs were incompetent and allowed retrograde filling of the microvasculature. Consequently, the number of valves/vein-density is decreased in venous ulcerated limbs. This finding may aid in understanding factors influencing the impact of reflux on the microcirculation in venous hypertension. 


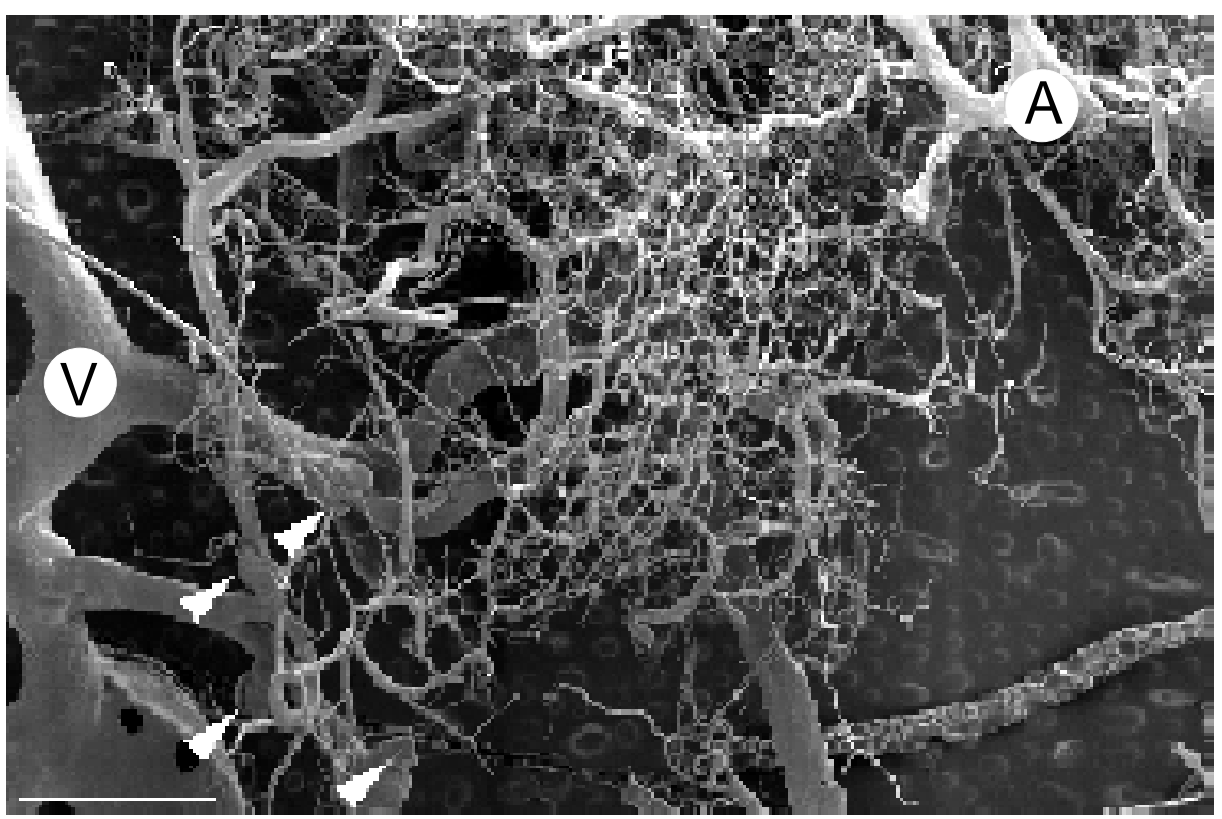

FIG 1 . The capillary bed networks associated with an artery (A) and vein (V). Four venous valves (arrowheads) are easily visible. Bar $=2 \mathrm{~mm}$.

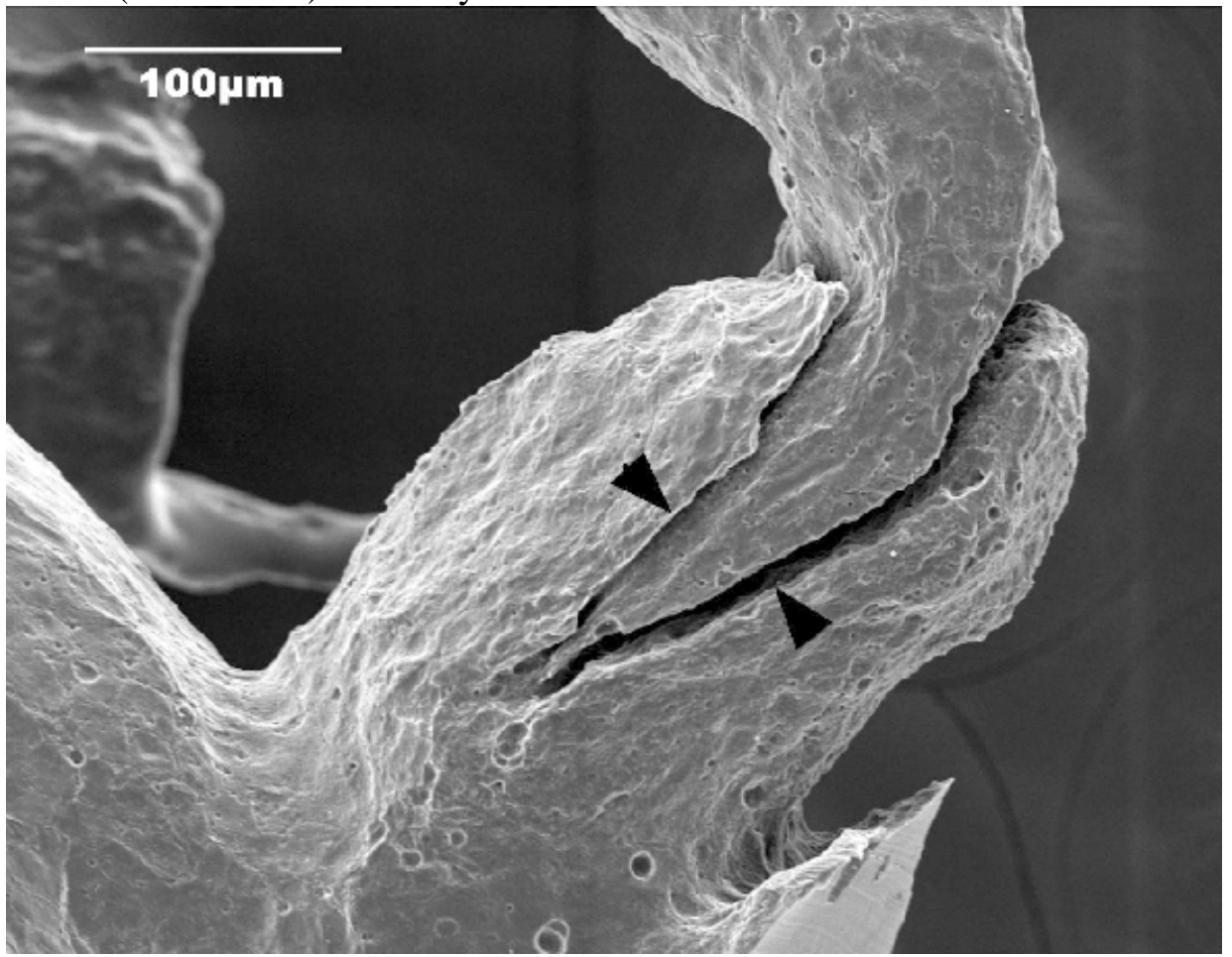

FIG 2. Example of the appearances of bicuspid venous valve identified by SEM of resin corrosion casts. A full venous valve cast, containing both the proximal and distal halves of the valve, joined by a small amount of resin passing between the cusps (arrow heads). Bar $=100 \mu \mathrm{m}$. 